\title{
Preguntas conceptuales para la enseñanza entre pares en Física Biológica
}

\section{Conceptual questions for peer teaching in Biological Physics}

DOI: $10.46814 /$ lajdv3n6-023

Recebimento dos originais: 01/11/2021

Aceitação para publicação: 14/12/2021

\author{
Daniel Alfredo Martínez \\ Escuela de Medicina Veterinaria y Profesorado de Nivel Medio y Superior en Biología \\ Sede Alto Valle y Valle Medio-Universidad Nacional de Río Negro. Pacheco 460, Choele Choel Río \\ Negro, Argentina (8360) \\ E-mail: dmartinez@unrn.edu.ar
}

\section{RESUMEN}

La metodología de enseñanza entre pares (EEP) ha sido aplicada con éxito en muchas universidades para la enseñanza de diversas disciplinas. Si bien se encuentran en la Web bases de datos con preguntas conceptuales utilizadas en esta metodología para la Física, la Química, la Biología, la Astronomía, las Geociencias y las Matemática, no se han desarrollado preguntas de este tipo para la Física Biológica. Se presentan aquí algunos ejemplos de preguntas conceptuales para esta disciplina aplicada. Las mismas están siendo empleadas en cursos de Física de los Procesos Biológicos de las carreras de Medicina Veterinaria y del Profesorado de Biología de la Universidad Nacional de Río Negro. Se observó que esta estrategia permite durante el desarrollo de los contenidos detectar dificultades en el aprendizaje y corregirlas. Por lo tanto se espera que a través de esta comunicación se difunda esta modalidad de enseñanza y surja la inquietud de que las preguntas conceptuales presentadas sean usadas en otras instancias educativas.

Palabras clave: Física Biológica, preguntas conceptuales, enseñanza entre pares.

\begin{abstract}
The peer teaching methodology (PTE) has been successfully applied in many universities for the teaching of various disciplines. Although databases with conceptual questions used in this methodology are found on the Web for Physics, Chemistry, Biology, Astronomy, Geosciences and Mathematics, no such questions have been developed for Biological Physics. Some examples of conceptual questions for this applied discipline are presented here. These questions are being used in Physics of Biological Processes courses in the Veterinary Medicine and Biology professorships at the National University of Río Negro. It was observed that this strategy allows detecting learning difficulties and correcting them during the development of the contents. Therefore, it is expected that through this communication this teaching modality will be disseminated and that the conceptual questions presented will be used in other educational instances.
\end{abstract}

Keywords: Biological Physics, conceptual questions, peer teaching. 


\section{INTRODUCCIÓN}

La metodología de enseñanza entre pares desarrollada por Eric Mazur (Crouch y Mazur, 2001) de la universidad de Harvard ha sido adoptada con éxito en otras universidades. La técnica consiste esencialmente en exposiciones breves de los temas de la clase, inmediatamente proponer una pregunta de elección múltiple sencilla referida al tema en cuestión, sobre la cual los alumnos trabajan inicialmente en forma individual, posteriormente se propone la discusión del problema en grupos, recolección de respuestas, y por último el profesor explica la respuesta correcta. La recolección de respuestas que puede realizarse en forma rápida contando manos levantadas para cada una de las opciones, permiten al docente determinar si el tema necesita una explicación más profunda o no, dependiendo del número de aciertos de las respuestas.

Se ha encontrado en la literatura trabajos referidos al empleo de la metodología de EEP, además de la Física, en áreas como la Biología y la Genética (Fagen 2003), la Química (Ellis et al, 2000; Landis et al, 2001), la Astronomía (Green, 2002), la Matemática (Terrell, 2005) y las Geociencias (Steer y McConnell, 2008). No se ha encontrado ningún trabajo de empleo de metodologías similares utilizadas en cursos de Física de los Procesos Biológicos que contemplen cuestiones de Física aplicada, por lo tanto, dado los éxitos logrados en la aplicación de esta técnica en otras áreas, es importante desarrollar preguntas propias y apropiadas a la EEP dentro del campo de la Física Biológica y promover la aplicación de esta técnica en el proceso de enseñanza aprendizaje de esta rama de la ciencia.

Se muestran aquí una serie de preguntas concernientes a la Física Biológica, algunas de las cuales se emplean en los cursos de Física Biológica y Física de los Procesos Biológicos de las carreras de Medicina Veterinaria y del Profesorado de Nivel Medio y Superior en Biología, ambas de la Universidad Nacional de Río Negro. Se describen también los resultados del empleo de esta técnica en los cursos de la UNRN que fueron indagados en forma exploratoria.

La presentación de este trabajo tiene como objetivos sociabilizar la metodología EEP, contribuir con ejemplos de preguntas conceptuales al desarrollo de propuestas similares, y comunicar los resultados de la implementación exploratoria realizada.

\section{EJEMPLOS DE PREGUNTAS CONCEPTUALES}

\subsection{EL CENTRO DE GRAVEDAD Y EL ANDAR DE LOS CUADRÚPEDOS}

La figura muestra con una cruz la posición del centro de gravedad (CG) de un equino con su cuatro patas (círculos llenos) apoyadas en el suelo. 




Si al andar levanta su pata delantera derecha (círculo blanco), ¿cuál de las siguientes figuras representa mejor la posición del nuevo centro de gravedad?

Imagen adaptada de Kane y Sternheim.1986. Física. Reverté, $2^{\text {da }}$ Edición. 702 pp.

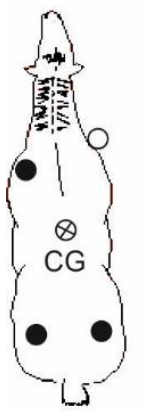

A

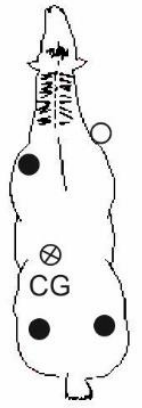

B



$\mathrm{C}$

\subsection{LEYES DE ESCALA Y LA DIVISIÓN CELULAR}

Una célula $(A)$ (supuesta esférica) tiene el doble de radio que otra $(B)$. La cantidad de oxígeno (y nutrientes) que necesita la célula $A$ es:



mayor que la $B$.

\subsection{LEYES DE ESCALA Y LA TASA METABÓLICA}

Un elefante es 200000 veces más pesado que un ratón. Para mantener su funciones vitales necesita:

$\square 10000 \square 200000 \square 300000 \square 400000$

veces más energía. 


\subsection{LEY DE LAPLACE Y LA PATOLOGÍA ALVEOLAR}

\subsection{LEY DE LAPLACE Y LA PATOLOGIA ALVEOLAR}

La figura muestra dos pompas formadas en las bocas del tubo que podrían representar dos alvéolos interconectados.

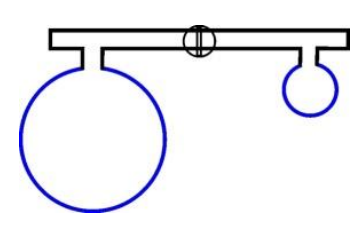

Al abrir la llave:



A: La pompa grande infla la chica quedando del mismo tamaño.



B: La pompa chica infla la grande.

\subsection{EL TEOREMA DE BERNOULLI Y LAS MADRIGUERAS}

La figura muestra una madriguera del perrito de las praderas que construye una de las entradas con una elevación para conseguir una circulación de aire en forma natural.



¿En qué sentido circulará el aire dentro de la madriguera?

\section{1. de $A$ hacia $B$}

2. de $B$ hacia $A$

\subsection{LEY DE POISEUILLE Y LA ARTERIOESCLEROSIS}

Por un tramo de arteria de longitud $L$ y diámetro $D$ circula sangre en régimen laminar (Fig 1)

Si el radio se reduce a la mitad (Fig 2), para mantener el caudal $Q$ constante, la presión debe aumentar: 


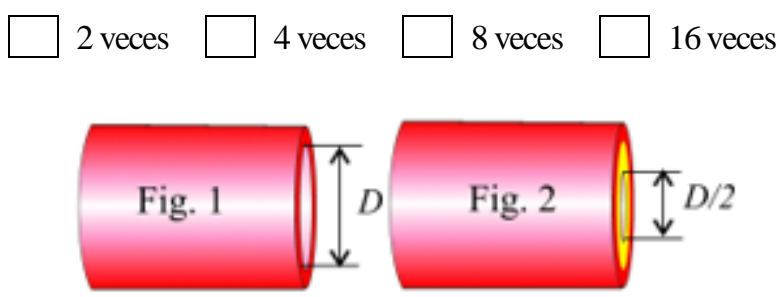

\subsection{RADIACIONES Y EL EFECTO INVERNADERO EN LA TIERRA}

El efecto invernadero es causado por:

a) La radiación ultravioleta al penetrar la capa de ozono.

b) La radiación infrarroja que es reemitida hacia la tierra por la capa de ozono.

c) La radiación infrarroja que es reemitida hacia la tierra por la atmósfera con bajos contenidos de ozono.

d) La radiación infrarroja que es reemitida hacia la tierra por la atmósfera con altos contenidos de $\mathrm{CO}_{2}$.

\section{8 ÓPTICA Y LOS DEFECTOS DE LA VISIÓN}

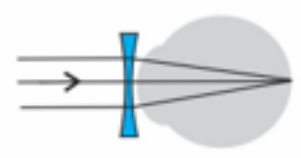

$A$

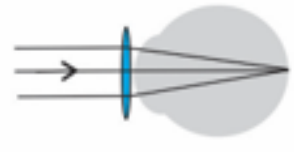

$B$

¿Cuál de las siguientes afirmaciones es correcta?

a) La lente de la figura $A$ se emplea para corregir la miopía pues sin ella la imagen se formaría delante de la retina.

b) La lente de la figura $B$ se emplea para corregir la miopía pues sin ella la imagen se formaría delante de la retina.

c) La lente de la figura $A$ se emplea para corregir la hipermetropía pues sin ella la imagen se formaría delante de la retina.

d) La lente de la figura $B$ se emplea para corregir la hipermetropía pues sin ella la imagen se formaría detrás de la retina.

\section{RESULTADOS DE LA INDAGACIÓNEXPLORATORIA}

La implementación de la técnica de EEP llevada a cabo en forma exploratoria en cursos de Física Biológica en la UNRN ha dado como resultado que:

1) La estrategia permite un rápido reconocimiento, a través de sus respuestas individuales, de las dificultades de los estudiantes en la comprensión de un contenido recientemente explicado. 
2) Las dificultades no son las mismas en cursos desarrollados en distintos años o asignaturas similares.

3) El intercambio de opiniones entre los alumnos facilita el cambio de respuestas individuales incorrectas a grupales correctas.

4) Un porcentaje importante de alumnos se abstiene de dar respuesta en forma individual a la pregunta conceptual formulada por el docente, lo que perjudica realizar un estudio cuantitativo de la estrategia EEP.

\section{CONCLUSIONES}

La estrategia de enseñanza entre pares, investigada en forma exploratoria en cursos de Física Biológica de la UNRN, permitió el reconocimiento de las dificultades de alumnos en el aprendizaje de un nuevo contenido, y de esta forma rever la forma de explicarlo nuevamente.

Se espera que a través de esta comunicación se difunda la modalidad de enseñanza entre pares y surja la inquietud de utilizar las preguntas presentadas en otras Instituciones. 


\section{REFERENCIAS}

Crouch, C. y Mazur, E. 2001. Peer Instruction: Ten years of experience and results, Am. J. Phys. 69 (9):970-977.

Ellis, A. B., Cappellari, A., Lisensky, G. C., Lorenz, J. K., Meeker, K., Moore, D., Campbell, K., Billmann, J., \& Rickert, K. 2000. ConcepTests.

Fagen, A. P. 2003. Assessing and Enhancing the Introductory Science Course in Physics and Biology: Peer Instruction, Classroom Demonstrations, and Genetics Vocabulary.

Green, P. 2002. Peer instruction for astronomy. Disponible en http://heawww.harvard.edu/ pgreen/educ/PIAhandout_25jan02.pdf consultada el 13 de diciembre de 2021.

Landis, C. R., Ellis, A. B., Lisensky, G. C., Lorenz, J. K., Meeker, K., \& Wamser, C. C. 2001. Chemistry ConcepTests: A pathway to interactive classrooms. Prentice Hall.

Steer, D. y McConnell, D. 2008. Starting point-Teaching entry level geoscience: ConcepTest examples. Disponible en: http://serc.carleton.edu/introgeo/interactive/ctestexm.html. consultada el 13 de diciembre de 2021.

Terrell, $2005 . \quad$ GoodQuestions Project. Disponible en: http://pi.math.cornell.edu/ maria/gq/handout2005.html consultada el 13 de diciembre de 2021. 